\title{
A SUPERVISED BAYESIAN APPROACH FOR SIMULTANEOUS SEGMENTATION AND CLASSIFICATION
}

\author{
Daniel C. Zanotta ${ }^{1}$, Matheus P. Ferreira ${ }^{2}$, Maciel Zortea ${ }^{3}$, Jean A. Espinozal, and Yosio Shimabukuro ${ }^{2}$ \\ 1 Institute for Education, Science and Technology - Rio Grande - RS - Brazil \\ 2 National Institute for Space Research - São José dos Campos - SP - Brazil \\ 3 Instituto de Informática - Universidade Federal do Rio Grande do Sul - Porto Alegre - RS - Brazil
}

\begin{abstract}
This paper presents a new paradigm for object based classification of multispectral images. Instead of classifying objects only after the segmentation process is completed, it is proposed to intercept the early stages of the segmentation by iteratively performing classification tests to under growing regions. By applying this simultaneous analysis, mislabeling of objects considered only after segmentation is completely done can be avoided. The proposed technique assumes that some growing regions can present higher membership to a particular class when comparing to the final object in which it is included. A Bayesian framework was applied in classification tests performed by pixel based, traditional object based, and the proposed technique were performed. The results show the soundness of the proposed method when comparing overall accuracies with a reference map.
\end{abstract}

Index Terms - image analysis, object based classification, Bayesian framework

\section{INTRODUCTION}

Object-based classification approaches have played a key role in remote sensing image analysis [1]. Depending on the specific application, this kind of classification provides great improvements when compared to pixel-based ones [2]. An important difference is that results achieved by object-based systems are more homogeneous, which makes analysis more convenient. Another advantage of dealing with regions instead of pixels is the possibility to add other attributes in the classification scheme, such as texture and shape. Despite these advantages, some applications showed that overall accuracy of object-based classifiers can be smaller than traditional pixel-based approaches [2]. In some segmented images, objects are not consistent with the pixels inside it, which lead to mislabeling in further classification processes. This situation can be caused by a phenomenon known as under segmentation, which results in too large regions. This occurs mainly when the user is prevented to appropriately set the segmentation parameters [3]. Unfortunately, the optimal choice of the parameters is generally difficult and arbitrary [4]. To overcome this situation, some image analysis techniques have been focusing on simultaneous process of segmentation and classification applied for signals [5], some kinds of medical images [6] and photo sequences [7]. Specifically for remote sensing, in [8] a statistical approach was tested to perform classification in growing regions trough a hypothesis testing. Comparison of this technique with the traditional segmentation followed by classification indicated slight improvement of the proposed approach. In this abstract, we suggest a new technique aimed at performing optimized supervised classification during segmentation process. In [8], exploratory experiments indicated the soundness of this kind of strategy for remote sensing images of natural scenes. In the present work, we suggest a Bayesian framework to early identify classes in under growth regions. Instead of using statistical tests for classification, the new methodology employs a parametric decision rule through the maximum likelihood classifier, thus not requiring minimum size of regions in order to early label them. By using this simultaneous methodology, growing regions with high class membership can be early associated to a given predefined class. Thus, avoiding eventual mislabeling caused by segmentation followed by classification. In order to optimize the process, regions can be previously labeled only if they have considerable degree of membership to one specific class in comparison with the others.

\section{PROPOSED CONCEPTUAL FRAMEWORK}

The proposed methodology can be seen as an interception into the segmentation process. Here, we use the region growing segmentation algorithm to test our formulation. Region growing algorithms are aimed to produce objects by iteratively merging pixels or regions in the image [9]. They start from the pixel level and apply similarity tests based on input parameters to determine whether two adjacent pixels must be merged or not. Depending on the specific region growing technique, the type and number of input parameters can vary. In this work, we implemented the region growing segmentation routine developed in [9]. A common stage to all region growing techniques is the comparison between adjacent regions in order to produce larger regions by merging them. The basis of the proposed methodology is to 
intercept this stage by submitting provisory regions to the classification test.

Let us consider a recently merged region $R$ with respective number of pixels $N_{R}$ and vector of means $\boldsymbol{\mu}_{R}$. As mentioned before, we propose to use the maximum likelihood classifier under the Bayes theorem to compare between $R$ and classes. As a supervised method, samples of each pre-defined classes are required. Using the samples, we can also derive the vector of means $\boldsymbol{\mu}_{i}$ and the covariance matrix $\boldsymbol{S}_{i}$ for each of the $n$ available classes $\omega i$. We assume to apply the proposed methodology only to natural scenes, where it is expected to have classes presenting Normal statistical distribution. Thus, the probability density function for each class $\omega i$, in respect to a given region $R$, is computed as

$$
G_{\omega i}(R)=\frac{1}{\sqrt{\left|\boldsymbol{S}_{i}\right|}} \cdot \exp \left\{-\frac{1}{2}\left[\left(\boldsymbol{\mu}_{R}-\boldsymbol{\mu}_{i}\right) \boldsymbol{S}_{i}^{-1}\left(\boldsymbol{\mu}_{R}-\boldsymbol{\mu}_{i}\right)^{t}\right]\right\}
$$

In our formulation, whenever two adjacent regions are merged, the resulting region $R$ will belong to a particular class $\omega c$ only if the following conditions are satisfied:

$$
\begin{aligned}
& N_{R} \geq T \\
& G_{\omega c}(R)>f \cdot G_{\omega i}(R) \quad \forall i \in[1, n]
\end{aligned}
$$

where $N_{R}$ is the number of pixels of $R, T$ is a user-defined threshold of size (number of pixels), $c$ is the analyzed class, $f$ is another user-defined factor indicating how many times $G_{c}$ $(R)$ have to be larger than $G_{i}(R)$ for the other classes. Factor $f$ can be seen as a coefficient which ensures the region will be labeled only if there is a high confidence to one of the classes compared to the others. The first condition verifies if the number of pixels of the region is large enough to label it. As stated before, in this formulation the size of the regions is not mandatory. However, if the user intends to obtain a classification map with more homogenous areas, the use of $T$ is encouraged.

The expected result is a partially classified segmented image. Regions not classified are those which have not satisfied the conditions in (2) for any of the available classes during the segmentation process, including the sub-regions gradually merged to form it. At the final step, the remaining unclassified regions are inserted into the traditional classification systems in order to complete the classification of the image.

\section{EXPERIMENT AND ANALYSIS}

In this study, we selected a TM-Landsat-5 subset $(310 \times 230$ pixels $)$ characterized by two types of soils and sparse vegetation. The image was acquired in October/2011 (path/row 221/82). A false color composition of the images is shown in Fig. 1a. This dataset was chosen because of the spectral similarity among targets, which is a challenge for classifiers. To assess the proposed technique, we used a reference map built up by visual interpretation and advanced knowledge about the region. Then, the dataset was classified by using the pixel-based, object-based traditional approaches, and the proposed technique. The region growing segmentation process performed here requires only two input parameters: similarity level and minimum region size. Here, we set them as 8 and 20, respectively. These parameters are default for deforestation applications using this segmentation algorithm [9]. For the proposed technique, which also requires two user-defined factors, we set 20 for $T$ and 10 for $f$. The rationale for using 20 for $T$ is that we look for results with the same minimum size of regions, allowing fair comparison among techniques. Factor $f$ was defined to ensure a considerable degree of membership when pre allocating growing regions to a particular class. We have taken three different samples corresponding to classes of vegetation, soil type A and soil type B. The reference map visually built for this experiment is showed in Fig. $1 \mathrm{~b}$.

The accuracy assessment was conducted by means of overall accuracy and per class accuracy. Table 1 summarizes the results reached by each technique. Controversially, the processing time spent by the proposed methodology was lower than the traditional segmentation process. This is explained by the fact that the time spent for iteratively classify the growing objects is offset by the reduction of the number of regions to be analyzed in the segmentation process.

Table 1. Accuracy assessment according to the reference map measured by overall accuracy.

\begin{tabular}{|c|c|c|c|}
\hline Technique & $\begin{array}{c}\text { Pixel } \\
\text { based }\end{array}$ & $\begin{array}{c}\text { Object } \\
\text { based }\end{array}$ & $\begin{array}{c}\text { Proposed } \\
\text { technique }\end{array}$ \\
\hline Sparse vegetation & $99.1 \%$ & $98.2 \%$ & $98.5 \%$ \\
\hline Soil type A & $73.2 \%$ & $72.2 \%$ & $78.2 \%$ \\
\hline Soil type B & $82.9 \%$ & $80.2 \%$ & $81.2 \%$ \\
\hline Overall Accuracy & $85.7 \%$ & $82.1 \%$ & $86.5 \%$ \\
\hline
\end{tabular}

Fig. 1c, 1d and 1e depict the resulting classification maps for the pixel-based, object-based, and proposed technique. As can be seen, the classification map achieved by the proposed technique shows more uniform areas, without the spatial noise commonly presented by pixel-based methods. In addition, some areas where the object-based classification show exceeded region merging, mainly between the two similar kinds of soil, were more appropriated classified by the proposed technique (Table 1). This fact corroborates our initial supposition that, by using the simultaneous segmentation and classification, eventual further mislabeling by classifying only after segmentation step is finished can be avoided. 


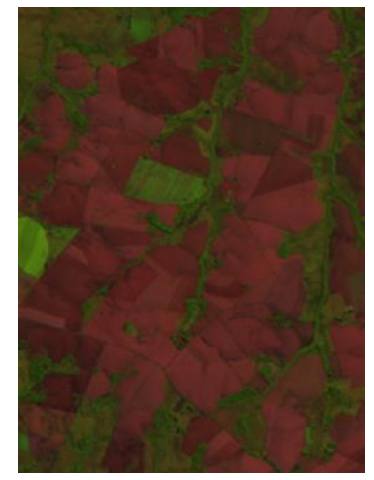

(a)

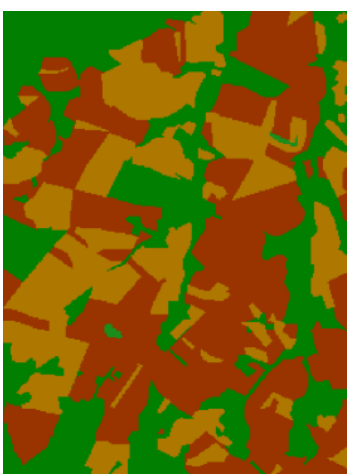

(b)

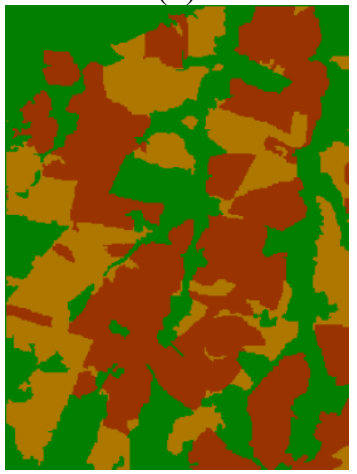

(d)

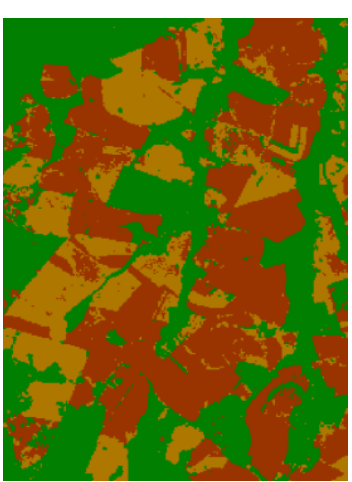

(c)

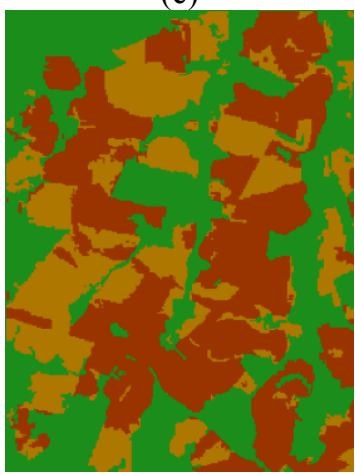

(e)
Fig. 1. (a) Multispectral TM-Landsat-5 image data employed in the experiment $(5(\mathrm{R}), 4(\mathrm{G}), 3(\mathrm{~B}))$; (b) reference map; classification results for (c) pixel-based approach, (d) object-based approach, and (e) proposed technique.

\section{CONCLUSIONS}

The results of the experiment indicate strength of the proposed methodology. Although the quantitative assessment has proved reasonable, the most important advantage of the proposed technique is that the user can obtain an appropriate level of pixel aggregation without losing classification accuracy. Moreover, since it is not based on statistical tests, there is no size limitation for growing segments to perform classification. This approach has showed more efficient to both special situations: (1) high spectral similarity of available classes and (2) low confidence in the segmentation input parameters.

\section{REFERENCES}

[1] T. Blaschke, G.J. Hay, M. Kelly, S. Lang, P. Hofmann, E. Addink, R. Queiroz Feitosa, F. van der Meer, H.van der Werff, and F. van Coillie, "Geographic object-based image analysis: Towards a new paradigm", ISPRS J. Photogramm. Remote Sens. vol. 87, pp. 180-191, 2014.

[2] C. Linli, S. Jun, T. Ping, and D. Huaqiang, "Comparison study on the pixel-based and object-oriented methods of landuse/cover classification with TM data", Earth Observation and Remote Sensing Applications, EORSA 2008. pp.1-5, June 30, 2008.

[3] M. Neubert, and G. Meinel. "Evaluation of segmentation programs for high resolution remote sensing applications", International ISPRS Workshop High resolution mapping from space, 2003.

[4] M. Jaesik, M.Powell, and K.W. Bowyer, "Automated performance evaluation of range image segmentation algorithms", Systems, Man, and Cybernetics, Part B: Cybernetics, IEEE Transactions on, vol: 34, no. 1, pp. 263 - 271, 2004.

[5] O. Cappé, "A Bayesian approach for simultaneous segmentation and classification of count data", IEEE Transactions on Signal Processing, vol. 5, no. 2, pp. 400-410, 2002.

[6] G. Lin, M.K. Chawla, K. Olson, C.A. Barnes, J.F. Guzowski, C. Bjornsson, W. Shain, and B. Roysam. "A multi-model approach to simultaneous segmentation and classification of heterogeneous populations of cell nuclei in 3D confocal microscope images". Cytometry A. vol. 71, no. 9, pp. 724-736, 2007.

[7] G. Laura, T. Jean-Philippe, and P. S. Nikos, "Space and Variational Methods in Computer Vision, A Variational Framework for the Simultaneous Segmentation and Object Behavior Classification of Image Sequences", Lecture Notes in Computer Science, vol. 4485, pp. 652-664, 2007.

[8] D.C. Zanotta, M.P. Ferreira, M. Zortea, and Y. Shimabukuro, "A statistical approach for simultaneous segmentation and classification," Geoscience and Remote Sensing Symposium (IGARSS), 2014 IEEE International, pp. 4899,4901, 2014.

[9] L. S. Bins, L. M. G. Fonseca, G. J. Erthal, and F. M. Ii, "Satellite imagery segmentation: a region growing approach". SBSR - Brazilian Remote Sensing Symposium, v. 8, pp. 677-680, 1996. 